\title{
Implications of Immunotherapy in Hepatobiliary Tumors
}

\author{
Aline Gottlieb Jan Best Ali Canbay \\ Department of Gastroenterology, Hepatology, and Infectious Diseases, University Hospital Magdeburg, \\ Otto von Guericke University Magdeburg, Magdeburg, Germany
}

\section{Keywords}

Hepatocellular carcinoma · Immunotherapy - Targeted therapy · Immune checkpoint inhibitors · Programmed cell death protein 1 antibody · Programmed death ligand 1 antibody · Cytotoxic T-lymphocyte-associated protein 4 antibody

\footnotetext{
Abstract

Hepatocellular carcinoma (HCC) remains one of the leading causes of cancer-related death worldwide. Upon ineligibility for resection, liver transplantation, or locoregional therapies, sorafenib has been the only systemic treatment option of advanced HCC for more than a decade. Immunotherapy is an evolving HCC treatment option that has shown promise in treatment efficacy at an acceptable safety profile during several preceding phase I/II trials. Numerous clinical trials of immune checkpoint inhibitors (ICPIs) alone, in combination of two, or combined with other targeted or locoregional therapies are ongoing. Encouraging results of two-phase III trials testing pembrolizumab or nivolumab versus standard care therapy even resulted in Food and Drug Administration approval for second-line treatment of advanced HCC. ICPIs may open new avenues to the treatment of hepatobiliary tumors, alone or in combination.

(C) 2019 S. Karger AG, Basel
}

\section{Introduction}

Hepatocellular carcinoma (HCC) remains one of the leading causes of cancer-related death worldwide $[1,2]$. Globally, HCC cases related to viral hepatitis are on the decline. In contrast, the incidence of HCC is rising, with hepatic steatosis seen as the major risk factor in the western world [1]. Despite the availability of numerous different therapeutic options, the 5-year overall survival (OS) in HCC patients averages $43 \%$ only [3].

For patients with advanced-stage HCC where curative treatment options (such as resection and liver transplantation) and locoregional therapies (i.e., radiofrequency or microwave ablation, transarterial chemoembolization) are no longer available, systemic treatment is indicated.

For more than a decade, sorafenib, a potent oral multityrosine kinase inhibitor (TKI) exhibiting antiproliferative and antiangiogenetic effects, was the only approved systemic HCC first-line treatment option (Fig. 1). In the phase III SHARP trial (NCT00105443), where sorafenib was tested against placebo, median OS was 10.7 months in the sorafenib arm and 7.9 months in the best supportive care arm [4]. In 2007, sorafenib was granted US Food and Drug Administration (FDA) priority review for HCC treatment based on the significant survival benefit demonstrated in the SHARP trial.

Ten years later, the phase III RESORCE (NCT01774344) trial tested the efficacy of the TKI regorafenib versus placebo for HCC patients who progressed on sorafenib treatment, resulting in improved OS by approximately 4 months compared to placebo $[5,6]$. Consecutively, regorafenib received FDA approval in April 2017 and later in the same year European Medicines Agency (EMA) approval for HCC second-line treatment (Fig. 1) [7].

In 2018, the results of the noninferiority designed phase III REFLECT trial (NCT01761266) were presented, testing the TKI lenvatinib against sorafenib in the firstline setting of advanced-stage HCC. The median OS time

\section{KARGER}

(C) 2019 S. Karger AG, Basel
Prof. Dr. med. Ali Canbay

Department of Gastroenterology, Hepatology, and Infectious Diseases

University Hospital Magdeburg, Otto von Guericke University Magdeburg

Leipziger Strasse 44, DE-39120 Magdeburg (Germany)

E-Mail ali.canbay@med.ovgu.de 
Fig. 1. Overview of FDA- and EMA-approved systemic HCC treatments. * In previously treated advanced HCC. EMA, European Medicines Agency; FDA, Food and Drug Administration; HCC, hepatocellular carcinoma.

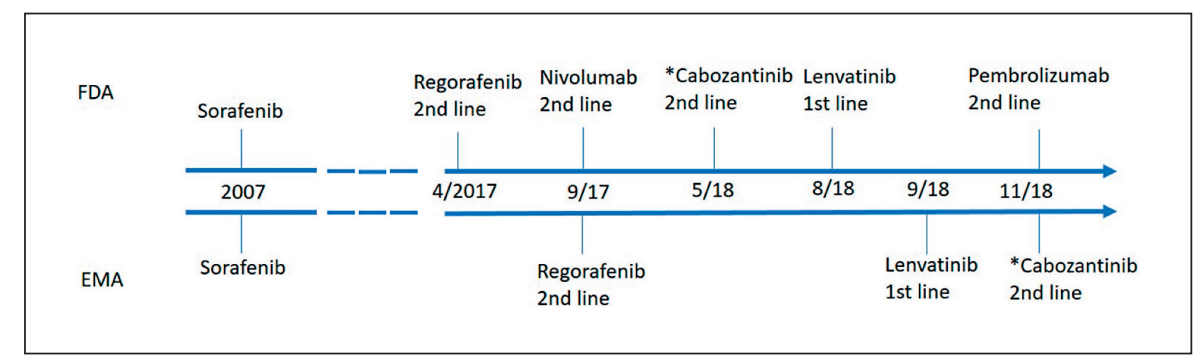

for lenvatinib of 13.6 months was noninferior to that of sorafenib (12.3 months), reaching its primary endpoint of noninferiority [8]. As a result, lenvatinib [9-11] was approved for first-line treatment of unresectable HCC by the Japanese Pharmaceuticals and Medical Devices Agency in March 2018, by the FDA in August 2018, and by the EMA right after (Fig. 1).

The recent phase III CELESTIAL trial (NCT01908426) evaluated cabozantinib as compared with placebo in previously sorafenib-treated patients with advanced HCC $[12,13]$. At the second planned interim analysis published in July 2018, median OS was 10.2 months with cabozantinib and 8.0 months with placebo (Fig. 1). As a result, treatment with cabozantinib showed significantly longer OS than placebo [14]. In May 2018, the FDA accepted cabozantinib application for advanced HCC, and EMA approval for second-line HCC treatment was received in November 2018.

Based on the results mentioned above, the recent European Association for the Study of the Liver (EASL) HCC guideline endorses sorafenib and lenvatinib $[15,16]$ as first-line systemic therapy of advanced HCC [17]. Regorafenib and cabozantinib (also both TKIs) are recommended for second-line treatment. However, all TKIs exhibit a broad spectrum of side effects, potentially impacting the patients' quality of life significantly. Therefore, it is mandatory to determine the safety and efficacy of immune checkpoint inhibitors (ICPIs) for the treatment of hepatobiliary tumors consecutively.

ICPIs enhance antitumor immunity by blocking tumor-induced immune suppression of cytotoxic T-cells, resulting in exaggerated immune activation. Lately, immune-based approaches have shown great promise in the treatment of various solid malignancies, having been associated with survival benefit and long-term disease control in melanoma, lung cancer, renal cell carcinoma, and other tumors [18].

The phase I/II CheckMate 040 trial (NCT01658878) and the phase II KEYNOTE-224 trial (NCT02702414), testing nivolumab and pembrolizumab, respectively in HCC patients pretreated with sorafenib, exhibited comparable overall response rates (for nivolumab 18.2\% per modified Response Evaluation Criteria in Solid Tumors
[mRECIST] and for pembrolizumab 17\% per Response Evaluation Criteria in Solid Tumors [RECIST] 1.1) [19, 20]. Based on those results, nivolumab received FDA approval in September 2017 and pembrolizumab in November 2018 (Fig. 1).

This review aims to summarize the most recent developments in standard of care systemic therapy of hepatobiliary tumors. Present and potential future implementation of immunotherapy in $\mathrm{HCC} /$ cholangiocarcinoma (CC) treatment will be highlighted in this context with a focus on clinical trials of ICPIs as either monotherapy or in combination with surgical, locoregional, or systemic targeted treatment options.

\section{Immunotolerance as a Promotor of Hepatocarcinogenesis}

Portal vein perfusion leads to continuous hepatic exposure to antigens from bacterial and environmental agents, requiring intrinsic tolerogenic mechanisms preventing permanent immune activation [21]. Furthermore, in the scenario of persistent hepatic inflammation, as it is the case in several viral or metabolic hepatopathies, liberation of proinflammatory cytokines and consecutive recruitment of immunosuppressive cells can aggravate intrinsic immunosuppression, which in turn can be used by tumor cells to evade detection [22]. Indeed, undetected tumor-associated antigens and other stimulants may promote tumor proliferation. Under physiological conditions, inhibitory or stimulatory signals are required to facilitate effective destructive response against pathogens and tumors on the one hand and to prevent an overwhelming immune response against normal tissue on the other hand.

T-cells play a pivotal role in cell-mediated tumor immunity and do so through counterbalance of costimulatory and coinhibitory cell-to-cell signals between different components of the immune system. Critical inhibitory and stimulatory checkpoint molecules control the immune system through regulation of this complex network. The inhibitory immune checkpoint proteins cytotoxic T-lymphocyte-associated protein 4 (CTLA-4) and 
programmed cell death protein $1(\mathrm{PD}-1)$ are receptors expressed on the surface of cytotoxic T-cells that interact with their cognate ligands CD80/CD86 and programmed death ligand 1 (PD-L1) on antigen-presenting cells, which helps the cancer cell evade T-cell-mediated death. Therefore, overexpression of PD-1, PD-L1, and CTLA-4 and consecutive $\mathrm{T}$-cell inhibition promotes tumor proliferation. In the case of HCC, PD-L1 overexpression is associated with more aggressive tumors and higher rates of postoperative recurrence [23].

\section{Inhibiting the Inhibitor}

Immunotherapies possess distinct mechanisms of action and primarily fall into the following categories: adoptive T-cell transfer, oncolytic viruses, cancer vaccines, and monoclonal antibodies [18]. It has been shown in numerous clinical HCC trials that monoclonal antibodies targeting checkpoint molecules have strong antitumor efficacy with an acceptable safety profile. Currently approved checkpoint inhibitors for several tumor entities are CTLA-4, PD-1, and PD-L1 inhibitors. ICPIs prevent receptors and ligands from binding to each other, thereby disrupting signaling [18] (and thus inhibiting the T-cell inhibition). Currently approved ICPIs for HCC treatment are the anti-PD-1 agents, nivolumab, and pembrolizumab.

\section{Potential Adverse Events}

In order to achieve sustained antitumor effect, "taking off the brakes" from the immune system might result in an unintended attack of healthy organ tissue as a side effect. Those are slightly different from other forms of targeted HCC therapy and require rapid and appropriate recognition and treatment. Although a majority of side effects are reversible, immediate and proper treatment is mandatory. With rapidly increasing use of ICPIs, it is imperative to be aware of common side effects including rash, diarrhea, lowering thyroid hormones, and fatigue. Furthermore, inflammation of the lung, intestines or liver, hormonal abnormalities, and kidney, heart, or neurologic problems are frequent. In case of most grade 2 toxicities, new guidelines from the American Society of Clinical Oncology (ASCO) and the National Comprehensive Cancer Network (NCCN) recommend suspension of ICPIs with consideration of resuming when symptoms revert to grade 1 or less. Grade 3 toxicities generally warrant suspension of ICPIs and the initiation of high-dose corticosteroids or even infliximab in some selected diseases. Unfortunately, steroid administration counteracts the antitumor effect of ICPIs. For grade 4 toxicities per- manent ICPI discontinuation is required, with the exception of endocrinopathies [24].

Administration of ICPIs in patients with preexisting autoimmune disease or history of organ transplantation demands thoughtful discussion of potential risks.

\section{Response Assessment of ICPIs}

RECIST facilitate radiological discrimination of therapy response (stable or disease progression) [25]. ICPI treatment exhibits distinct differences in response assessment compared to targeted or locoregional HCC therapy. Conventional RECIST 1.1 or mRECIST may underestimate the benefit of immunotherapeutics, since the effect of ICPIs is a multistep process (cellular response, then antitumor response, and finally treatment response). Consequently, antitumor response to immunotherapy may be significantly longer compared to that of targeted therapies [26]. To address this issue, immune RECIST (iRECIST) were developed, which provide better assessment of the effect of therapy [27]. Here, the bar will be reset if RECIST progressive disease is followed at the next timepoint by tumor shrinkage. The new overall response is defined as immune unconfirmed progressive disease. It should be considered that, in absence of clinical progression, pseudoprogression on radiographic imaging patients has to be reevaluated carefully to prevent too early ICPI discontinuation.

\section{PD-1 Inhibitors}

\section{Nivolumab}

Nivolumab is a fully human monoclonal antibody targeting PD-1. It was first tested in HCC patients during the CheckMate 040 trial, with promising results, published in 2017 [19]. In this phase I/II study, it was administered to patients who progressed on prior sorafenib treatment or those who exhibited intolerable toxicity. The primary endpoint was amongst safety studies the objective response rate (ORR). Approximately $40 \%$ of patients enrolled had either stable disease or showed partial response. Based on those findings, in the same year the FDA approved nivolumab for patients who had been pretreated with sorafenib. The phase III CheckMate 459 (NCT02576509) trial is active, but not recruiting anymore, comparing nivolumab versus sorafenib as first-line therapy; results are expected for 2019. The primary endpoint is OS. Furthermore, the CheckMate 9DX (NCT03383458) phase III study examines the efficacy of nivolumab in HCC patients with a high risk of recurrence after partial hepatectomy or ablation against placebo. The study has been recruiting since December 2017. The primary endpoint is recurrence-free survival. 


\section{Pembrolizumab}

Pembrolizumab (MK-3475) is another monoclonal PD-1 antibody, which was studied in a phase II, openlabel trial (KEYNOTE-224) [20]. In this study, patients with advanced HCC progression or intolerance to sorafenib, or patients naive for systemic therapy, were enrolled. Patients received $200 \mathrm{mg}$ pembrolizumab every 3 weeks. The primary endpoint was ORR. A total of 104 patients were enrolled, of whom 1 showed complete response, 17 partial response, and 46 stable disease; 34 patients had progressive disease. In summary, the disease in $61.5 \%$ of the participants was controlled. The median time to response was 2.1 months, with a median duration of response (DOR) of 8 months. Interestingly, the authors showed a different response in target lesions depending on the etiology of the HCC. Indeed, viral hepatitis-based HCC might need a different therapy than alcohol-based HCC. The data on OS and progression-free survival (PFS) are encouraging. The most common adverse events were fatigue, diarrhea, pruritus, and an elevation in aspartate aminotransferase. In 1 case the treatment accounted for death of the patient. Currently, a phase III trial is ongoing (MK-3475-671/KEYNOTE-671, NCT03425643), testing pembrolizumab versus standard of care systemic therapy or placebo. The primary outcomes are event-free survival and OS [20].

\section{CTLA-4 Inhibitors}

CTLA-4, a protein receptor functioning as an immune checkpoint, downregulates immune responses. CTLA-4 is constitutively expressed in regulatory T-cells, but only upregulated in conventional T-cells after activation, a phenomenon which is particularly notable in cancers [28]. Despite its positive antitumor activity, patients specifically treated with CTLA- 4 antibodies or in combination with checkpoint-blocking antibodies are at higher risk of immune-related adverse events (compared to the occurrence of adverse side effects in the treatment with PD-1 inhibitors), such as dermatologic, gastrointestinal, endocrine, or hepatic autoimmune reactions [29].

\section{Tremelimumab}

Tremelimumab (formerly ticilimumab, CP-675,206) is a human monoclonal antibody against CTLA-4. Tremelimumab was first tested in a phase II trial (NCT01008358) in a small HCC cohort of 20 sorafenib-naive or -experienced patients with chronic hepatitis $C$ virus infection [30]. Despite promising response rate and partial response according to RECIST, time to progression (TTP) (6.48 months, 95\% CI 3.95-9.14) as well as OS (10.7 vs. 11.7 months) were disappointing; potentially this has to be attributed to the inclusion of 9 Child-Pugh B patients.
Later, a combination phase I/II trial tested tremelimumab and durvalumab versus sorafenib. The phase I trial (NCT02978482) is finished, and promising results were presented at the ASCO annual meeting 2018 by Kelley et al. [31]. Phase II is still ongoing (NCT02519348), and a phase III trial (HIMALAYA, NCT03298451) also combining durvalumab with tremelimumab versus sorafenib in the first-line setting started recruiting in October 2018 (see also Durvalumab).

\section{Ipilimumab}

Ipilimumab, a monoclonal antibody targeting CTLA4, showed promising results in melanoma treatment [32] and was likely to show a positive effect in HCC patients as well. Indeed, it has been demonstrated that CTLA-4 treatment increased the T-cells' antitumor response [33]. Notably, there is a phase II trial registered from the beginning of 2018, studying neoadjuvant effects of nivolumab plus ipilimumab (NCT03510871) in estimated 40 patients; recruitment start is pending. The study objective will be to evaluate efficacy in terms of tumor shrinkage, ORR, downstaging rate, and safety profile and to concordantly collect HCC tumor tissue and peripheral blood samples from the patients for a comprehensive biomarker evaluation for nivolumab plus ipilimumab immunotherapy. The primary endpoint will be the percentage of subjects with tumor shrinkage (according to the RECIST criteria).

\section{PD-L1 Inhibitors}

\section{Atezolizumab}

Atezolizumab is a fully humanized, engineered monoclonal antibody against PD-L1 [34]. Interim analysis data of a phase I trial, testing atezolizumab in combination with bevacizumab versus different standard chemotherapy regimens (NCT02715531), was presented at the ASCO annual meeting 2018. The primary endpoints are PFS and objective response (according to the iRECIST criteria). This study showed at the time of the data cutoff (October 24, 2017) that 26 patients were evaluable for safety and confirmed that partial responses occurred in 13 patients (62\%) regardless of HCC etiology, and region (Asia or US); the median estimates for PFS, DOR, TTP, and OS have not yet been reached [35]. The trial will be recruiting until May 2019.

\section{Avelumab}

Avelumab is also a human monoclonal antibody targeting PD-L1. Two trials are currently ongoing and are recruiting actively. One is testing the effectiveness of avelumab in combination with axitinib in HCC patients in a phase II trial (VEGF Liver 100, NCT03289533). The primary endpoint is the assessment of adverse events. Another phase II trial tests avelumab in patients with ad- 
Table 1. Selected ICPI trials in combination with locoregional/targeted therapy

\begin{tabular}{|c|c|c|c|c|}
\hline Study & NCT ID & $\begin{array}{l}\text { Partici- } \\
\text { pants }\end{array}$ & Phase & $\begin{array}{l}\text { Estimated } \\
\text { completion } \\
\text { date }\end{array}$ \\
\hline
\end{tabular}

Trials combining local therapies and ICPIs

\begin{tabular}{|c|c|c|c|c|}
\hline Study of Y-90 radioembolization with nivolumab in Asians with HCC & NCT03033446 & 40 & II & 2020 \\
\hline $\begin{array}{l}\text { Study of the safety and antitumoral efficacy of nivolumab after SIRT for the } \\
\text { treatment of patients with HCC (NASIR-HCC) }\end{array}$ & NCT03380130 & 40 & II & 2019 \\
\hline $\begin{array}{l}\text { TACE in combination with nivolumab performed for intermediate-stage } \\
\text { HCC (IMMUTACE) }\end{array}$ & NCT03572582 & 49 & II & 2022 \\
\hline $\begin{array}{l}\text { Study to test the safety and feasibility of nivolumab with drug-eluting bead } \\
\text { TACE in patients with liver cancer }\end{array}$ & NCT03143270 & 14 & I & 2019 \\
\hline
\end{tabular}

\section{Inhibitors and targeted therapy}

FGF401 in HCC and solid tumors characterized by positive FGFR4 and KLB NCT02325739 expression (+/- PDR001)

172 FGF401: I; 2020

FGF401 plus PDR001: II

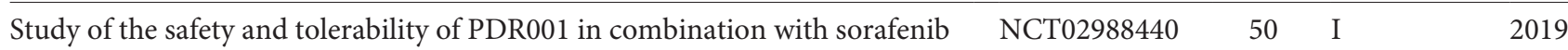
and to identify the maximum tolerated dose and/or phase II dose for this combination in advanced HCC patients

\section{ICPI and ICPI}

Study of durvalumab and tremelimumab as first-line treatment in patients with unresectable HCC (HIMALAYA)

FGF401, FGFR4 inhibitor; HCC, hepatocellular carcinoma; ICPI, immune checkpoint inhibitor; NCT, National Clinical Trial; PDR001, anti-programmed cell death protein 1 antibody; SIRT, selective internal radiotherapy; TACE, transarterial chemoembolization.

vanced HCC after prior sorafenib treatment (Avelumab HCC, NCT03389126). The primary endpoint in this study is the response rate (according to RECIST 1.1).

\section{Durvalumab}

Durvalumab (MEDI4736) is a human IgG1к monoclonal anti-PD-L1 antibody that blocks the interaction of PDL1 with the PD-1 and CD80 (B7.1) molecules. It was already tested in 40 patients with unresectable HCC, the majority of them having been pretreated with sorafenib [36]. Since this phase I/II study showed promising PFS and OS at an acceptable safety profile [37], the decision was made to test it in combination with tremelimumab (HIMALAYA). This phase III trial tests durvalumab and durvalumab plus tremelimumab against sorafenib. The primary endpoint is OS. First data were published at the ASCO meeting 2018, showing no unexpected safety signals for durvalumab and tremelimumab in this population.

\section{Combination Trials}

Clinical trials combining different ICPIs or targeted therapies with CPIs might reveal potential future synergisms for HCC treatment. It was intriguing to combine locoregional treatment with antiangiogenetic agents or antibodies. However, trials where transarterial chemoembolization was combined with sorafenib [38-40], brivanib [41], and orantinib [42] did not reach their primary endpoint of improving OS. Therefore, it will be crucial to discover whether ICPIs in combination with locoregional therapy will exhibit better synergism. Table 1 provides an overview of ongoing combination trials.

\section{Lenvatinib and Pembrolizumab}

At the ASCO annual meeting 2018, a combination phase Ib study was presented where patients were treated with lenvatinib and pembrolizumab (NCT03006926). The primary endpoints are dose-limiting toxicity and adverse events, and the extended primary endpoints are ORR and DOR. Of the 30 patients enrolled (part 1: patients not eligible for other therapies, $n=6$; part 2: patients with no prior therapy, $n=20$ ), 23 are still undergoing treatment (part 1: $n=3$; part 2: $n=20$ ). At the time of presentation (data cutoff March 2018, beginning of recruitment was February 2017), no patient showed progressive disease, but there were three fatal adverse events: two deaths were considered to be treatment-related and one case was not considered to be treatment-related. The 
Table 2. Selection of some target therapies for CC

\begin{tabular}{|c|c|c|c|}
\hline Study & NCT ID & $\begin{array}{l}\text { Estimated } \\
\text { participants }\end{array}$ & Phase \\
\hline \multicolumn{4}{|l|}{ IDH inhibitors } \\
\hline Phase I study of BAY 1436032 in patients with IDH1-mutant solid tumors & NCT02746081 & 81 & I \\
\hline $\begin{array}{l}\text { Study of AG-120 (ivosidenib) in previously treated advanced CC with IDH1 mutations } \\
\text { (ClarIDHy) }\end{array}$ & NCT02989857 & 186 & III \\
\hline \multicolumn{4}{|l|}{ FGFR inhibitors } \\
\hline Study of TAS-120 in patients with advanced solid tumors & NCT02052778 & $\mathrm{n} / \mathrm{a}$ & I \\
\hline Phase II, single-arm study of BGJ398 in patients with advanced CC & NCT02150967 & 120 & II \\
\hline $\begin{array}{l}\text { Study to evaluate the clinical efficacy of JNJ- } 42756493 \text { (erdafitinib), a pan-FGFR TKI, } \\
\text { in Asian participants with advanced non-small-cell lung cancer, urothelial cancer, } \\
\text { esophageal cancer, or CC }\end{array}$ & NCT02699606 & $\mathrm{n} / \mathrm{a}$ & II \\
\hline $\begin{array}{l}\text { Study to evaluate the efficacy and safety of pemigatinib versus chemotherapy in } \\
\text { unresectable or metastatic CC (FIGHT-302) }\end{array}$ & NCT03656536 & 432 & III \\
\hline \multicolumn{4}{|l|}{ Others } \\
\hline $\begin{array}{l}\text { Phase I study to evaluate the safety, pharmacokinetics, and pharmacodynamics of } \\
\text { H3B-6527 in participants with advanced HCC }\end{array}$ & NCT02834780 & 128 & I \\
\hline Trastuzumab in HER2-positive biliary tract cancer (BILHER) & NCT03613168 & 15 & II \\
\hline $\begin{array}{l}\text { Basket study of entrectinib (RXDX-101) for the treatment of patients with solid tumors } \\
\text { harboring NTRK 1/2/3 (Trk A/B/C), ROS1, or ALK gene rearrangements (fusions) } \\
\text { (STARTRK-2) }\end{array}$ & NCT02568267 & 300 & II \\
\hline Bortezomib in intrahepatic CC & NCT03345303 & 50 & III \\
\hline
\end{tabular}

CC, cholangiocarcinoma; FGFR, fibroblast growth factor receptor; IDH, isocitrate dehydrogenase; $n$ /a, not available; NCT, National Clinical Trial; TKI, tyrosine kinase inhibitor.

ORR ranged between 88 and 95\% (part 1) and between 43 and 59\% (part 2), depending on whether unconfirmed responses were included. These presented data showed noninferiority. The conductors of the study have amended to enroll 94 patients to part 2 to confirm the efficacy and safety data observed so far.

\section{FGF401 and PDR001}

FGF401 is an FGFR4 inhibitor which is tested as monotherapy versus in combination with PDR001, a humanized anti-PD-1 IgG4 antibody, in a multicenter phase I and II study (NCT02325739). The primary outcomes are ORR and TTP. Preliminary data were published in 2017, showing promising results considering adverse effects. It has also been discussed that FGF19 might be a possible future prognostic marker [43].

\section{Selective Internal Radiotherapy and ICPIs}

Tumor-immune cell interactions play a pivotal role for patient survival, and there is increasing evidence that locoregional therapies (e.g., selective internal radiotherapy [SIRT]) may be successfully combined with cancer im- munotherapies targeting ICPIs [44]. In 2017, a phase II multicenter trial on SIRT patients treated with nivolumab was launched and is still recruiting (NCT03380130). It was hypothesized that nivolumab may improve the antitumor effect of SIRT by enhancing antitumor immune responses. Indeed, it has recently been shown that SIRT impairs cellular immune function [45]. Thus, counteracting the immunosuppressive effect of SIRT by immunotherapy may enhance tumor control and therefore may be beneficial.

\section{Individualized Medicine and Potential Biomarkers in HCC}

The molecular diversity of HCC is well established [46], and it is characterized by an average of 30-40 mutations per tumor which may either derive from different cells of origin or from the activation of different oncogenic pathways $[38,39]$. For the latter, several studies even proposed subclasses based on gene expression profiling [40-42]. 
In the past, the majority of systemic treatment approaches have been focused on a limited number of targets despite HCC's molecular heterogeneity. To address this, a more biomarker-driven therapy stratification is mandatory in the future. However, the identification of a single biomarker predicting antiproliferative potential of targeted therapies remains challenging [47].

Recently, the role of alpha-fetoprotein (AFP) as a biomarker for treatment stratification was demonstrated during a phase III trial (REACH, NCT01140347) in HCC patients treated with ramucirumab, a monoclonal antibody targeting VEGF R2 versus placebo [43]. The outcome for ramucirumab was disappointing, showing no significant OS benefit [48, 49]; however, a subset analysis suggested potential benefits for patients with an initial high AFP level (>400 ng/mL). Based on those findings, a follow-up phase III trial (REACH-2, NCT02435433) selectively enrolled patients with AFP $>400 \mathrm{ng} / \mathrm{mL}$. First data were presented at the ASCO annual meeting 2018, showing a significantly superior OS (8.5 vs. 7.3 months) in this subset treated with ramucirumab compared to placebo. Taken together, this was the first trial demonstrating the superiority of biomarker-based patient selection.

Also in the case of ICPI treatment, for numerous tumor entities, PD-L1 expression can be utilized as a prognostic marker, and it correlates with a therapeutic response [50]. However, studies aiming to correlate PD-L1 expression and response prediction on ICPI treatment in HCC patients have failed to facilitate treatment stratification [51]. This might be due to a great variety of PD-L1 expression in HCC [52].

Another general issue of note is the molecular profile on a single tumor biopsy: there is no guarantee that the biopsy represents the entire tumor tissue [53]. Furthermore, the activation of a certain cascade or the expression of a target do not imply a definite tumor dependency [46].

\section{Systemic Therapy in CC}

Among other epithelial cancers, CC exhibits a very poor prognosis and limited systemic treatment options [54]. It can be divided into intrahepatic, perihilar, and distal CC [55]. For a long time, these three tumors were treated as one entity, despite the fact that each has its own pathogenesis and clinical outcome [55]. Up to date, an efficient targeted therapy for intrahepatic CC has not been found yet, the standard of care systemic therapy being chemotherapy with gemcitabine and cisplatin.

Despite numerous clinical trials being conducted with molecular targeted agents, including erlotinib, cetuximab, panitumumab, bevacizumab, sorafenib, cediranib, trametinib, and vandetanib, no agent has been shown to be effective for advanced biliary tract cancer [56]. In con- trast to HCC, in intrahepatic CC, PD-L1 expression can be considered a prognostic factor [57]. Table 2 provides an overview of ongoing clinical trials in CC. Substances such as LDK378 (ceritinib), an anaplastic lymphoma kinase inhibitor, and BKM120, a phosphoinositide 3-kinase inhibitor, were not able to meet their primary endpoint, and the trials were terminated ahead of time.

\section{Conclusion}

During the past decade, systemic treatment approaches for advanced-stage HCC were mainly based on targeted therapies with multi-TKIs, such as sorafenib. Recently, the inhibition of immune checkpoint regulators has restructured the treatment algorithms of several other cancer entities. In particular, the application of ICPI treatment for $\mathrm{HCC}$ is quickly evolving, since recent phase III trials clearly demonstrated that in some cases deep and durable responses are achievable at an acceptable safety profile. Those findings resulted in FDA approval for nivolumab and pembrolizumab in HCC treatment. Numerous trials of ICPIs in combination with other systemic or locoregional treatments are ongoing, potentially offering new hope for HCC patients. Further translational and clinical research is mandatory to identify potential biomarkers predicting response to immunotherapies as an integrative part of future personalized therapy concepts.

\section{Statement of Ethics}

The authors have no ethical conflicts to disclose.

\section{Disclosure Statement}

A. Gottlieb, A. Canbay: no conflicts of interest to declare. J. Best: consultant for Biocompatibles UK, Eisai, BMS; speaker honoraria: Biocompatibles UK, Abbvie. No funding was received.

References 1 Ferlay J, Soerjomataram I, Dikshit R, Eser S, Mathers C, Rebelo M, et al. Cancer incidence and mortality worldwide: sources, methods and major patterns in GLOBOCAN 2012. Int J Cancer. 2015 Mar;136(5):E359-86.

2 Bray F, Ferlay J, Soerjomataram I, Siegel RL, Torre LA, Jemal A. Global cancer statistics 2018: GLOBOCAN estimates of incidence and mortality worldwide for 36 cancers in 185 countries. CA Cancer J Clin. 2018 Nov;68(6): 394-424.

3 Tian G, Yang S, Yuan J, Threapleton D, Zhao $\mathrm{Q}$, Chen F, et al. Comparative efficacy of treatment strategies for hepatocellular carcinoma: systematic review and network meta-analysis. BMJ Open. 2018 Oct;8(10):e021269. 
4 Llovet JM, Ricci S, Mazzaferro V, Hilgard P, Gane E, Blanc JF, et al.; SHARP Investigators Study Group. Sorafenib in advanced hepatocellular carcinoma. N Engl J Med. 2008 Jul; 359(4):378-90.

5 Bruix J. Regorafenib and the RESORCE trial: a new second-line option for hepatocellular carcinoma patients. Hepat Oncol. 2016 Aug; 3(3):187-9.

6 Bruix J, Qin S, Merle P, Granito A, Huang YH, Bodoky G, et al.; RESORCE Investigators. Regorafenib for patients with hepatocellular carcinoma who progressed on sorafenib treatment (RESORCE): a randomised, double-blind, placebo-controlled, phase 3 trial. Lancet. 2017 Jan;389(10064):56-66.

7 Bruix J, Tak WY, Gasbarrini A, Santoro A, Colombo M, Lim HY, et al. Regorafenib as second-line therapy for intermediate or advanced hepatocellular carcinoma: multicentre, open-label, phase II safety study. Eur J Cancer. 2013 Nov;49(16):3412-9.

8 Kudo M, Finn RS, Qin S, Han KH, Ikeda K, Piscaglia $\mathrm{F}$, et al. Lenvatinib versus sorafenib in first-line treatment of patients with unresectable hepatocellular carcinoma: a randomised phase 3 non-inferiority trial. Lancet. 2018 Mar;391(10126):1163-73.

9 Kudo M: Lenvatinib may drastically change the treatment landscape of hepatocellular carcinoma. Liver Cancer. 2018 Mar;7(1):1-19.

10 Kudo M: Extremely high objective response rate of lenvatinib: its clinical relevance and changing the treatment paradigm in hepatocellular carcinoma. Liver Cancer. 2018 Sep; 7(3):215-24.

11 Kudo M: Lenvatinib in advanced hepatocellular carcinoma. Liver Cancer. 2017 Nov;6(4): 253-63.

12 Abou-Alfa GK, Borgman-Hagey AE, Kelley RK. Cabozantinib in Hepatocellular Carcinoma. N Engl J Med. 2018 Oct;379(14):1384-5.

13 Abou-Alfa G. Cabozantinib: targeted therapy back to the future? Ann Oncol. 2017 Mar; 28(3):450-1.

14 Abou-Alfa GK, Meyer T, Cheng AL, ElKhoueiry AB, Rimassa L, Ryoo BY, et al. Cabozantinib in Patients with Advanced and Progressing Hepatocellular Carcinoma. N Engl J Med. 2018 Jul;379(1):54-63.

15 Ikeda M, Okusaka T, Mitsunaga S, Ueno $\mathrm{H}$ Tamai T, Suzuki T, et al. Safety and Pharmacokinetics of Lenvatinib in Patients with Advanced Hepatocellular Carcinoma. Clin Cancer Res. 2016 Mar;22(6):1385-94

16 Ikeda K, Kudo M, Kawazoe S, Osaki Y, Ikeda M, Okusaka T, et al. Phase 2 study of lenvatinib in patients with advanced hepatocellular carcinoma. J Gastroenterol. 2017 Apr;52(4): $512-9$.

17 European Association for the Study of the Liver. Electronic address: easloffice@easloffice.eu; European Association for the Study of the Liver. EASL Clinical Practice Guidelines: Management of hepatocellular carcinoma. J Hepatol. 2018 Jul;69(1):182-236.

18 Dine J, Gordon R, Shames Y, Kasler MK, Barton-Burke M. Immune Checkpoint Inhibitors: An Innovation in Immunotherapy for the Treatment and Management of Patients with Cancer. Asia Pac J Oncol Nurs. 2017 Apr-Jun;4(2):127-35.
19 El-Khoueiry AB, Sangro B, Yau T, Crocenzi TS, Kudo M, Hsu C, et al. Nivolumab in patients with advanced hepatocellular carcinoma (CheckMate 040): an open-label, noncomparative, phase $1 / 2$ dose escalation and expansion trial. Lancet. 2017 Jun;389(10088): 2492-502.

20 Zhu AX, Finn RS, Edeline J, Cattan S, Ogasawara S, Palmer D, et al.; KEYNOTE-224 investigators. Pembrolizumab in patients with advanced hepatocellular carcinoma previously treated with sorafenib (KEYNOTE-224): a non-randomised, open-label phase 2 trial. Lancet Oncol. 2018 Jul;19(7):940-52.

21 Knolle PA, Thimme R. Hepatic immune regulation and its involvement in viral hepatitis infection. Gastroenterology. 2014 May; 146(5):1193-207.

22 Makarova-Rusher OV, Medina-Echeverz J, Duffy AG, Greten TF. The yin and yang of evasion and immune activation in HCC. J Hepatol. 2015 Jun;62(6):1420-9.

23 Gao Q, Ye F, Xia X, Xing H, Lu Y, Zhou J, et al. Correlation between PTEN expression and PI3K/Akt signal pathway in endometrial carcinoma. J Huazhong Univ Sci Technolog Med Sci. 2009 Feb;29(1):59-63.

24 Brahmer JR, Lacchetti C, Schneider BJ, Atkins MB, Brassil KJ, Caterino JM, et al.; National Comprehensive Cancer Network. Management of Immune-Related Adverse Events in Patients Treated With Immune Checkpoint Inhibitor Therapy: American Society of Clinical Oncology Clinical Practice Guideline. J Clin Oncol. 2018 Jun;36(17):1714-68.

25 Gavanier M, Ayav A, Sellal C, Orry X, Claudon $\mathrm{M}$, Bronowicki JP, et al. CT imaging findings in patients with advanced hepatocellular carcinoma treated with sorafenib: Alternative response criteria (Choi, European Association for the Study of the Liver, and modified Response Evaluation Criteria in Solid Tumor (mRECIST)) versus RECIST 1.1. Eur J Radiol. 2016 Jan;85(1):103-12.

26 Kantarci M, Pirimoglu B. Radiological Response to the Locoregional Treatment in Hepatocellular Carcinoma: RECIST, mRECIST, and Others. J Gastrointest Cancer. 2017 Jun; 48(3):282-5.

27 Seymour L, Bogaerts J, Perrone A, Ford R, Schwartz LH, Mandrekar S, et al.; RECIST working group. iRECIST: guidelines for response criteria for use in trials testing immunotherapeutics. Lancet Oncol. 2017 Mar; 18(3):e143-52.

28 Syn NL, Teng MW, Mok TS, Soo RA. De-novo and acquired resistance to immune checkpoint targeting. Lancet Oncol. 2017 Dec; 18(12):e731-41

29 Postow MA, Callahan MK, Wolchok JD. Immune Checkpoint Blockade in Cancer Therapy. J Clin Oncol. 2015 Jun;33(17):1974-82.

30 Sangro B, Gomez-Martin C, de la Mata M, Iñarrairaegui M, Garralda E, Barrera $P$, et al. A clinical trial of CTLA-4 blockade with tremelimumab in patients with hepatocellular carcinoma and chronic hepatitis C. J Hepatol. 2013 Jul;59(1):81-8.
31 Kelley RK, Abou-Alfa GK, Bendell JC, Kim TY, Borad MJ, Yong WP, et al. Phase I/II study of durvalumab and tremelimumab in patients with unresectable hepatocellular carcinoma (HCC): phase I safety and efficacy analyses. J Clin Oncol. 2017:suppl abstr 4073.

32 Hodi FS, O’Day SJ, McDermott DF, Weber RW, Sosman JA, Haanen JB, et al. Improved survival with ipilimumab in patients with metastatic melanoma. N Engl J Med. 2010 Aug;363(8):711-23.

33 Mizukoshi E, Nakamoto Y, Arai K, Yamashita T, Sakai A, Sakai Y, et al. Comparative analysis of various tumor-associated antigen-specific $\mathrm{T}$-cell responses in patients with hepatocellular carcinoma. Hepatology. 2011 Apr; 53(4):1206-16

34 Brower V. Anti-PD-L1 antibody active in metastatic bladder cancer. Lancet Oncol. 2015 Jan;16(1):e11.

35 Stein S, Pishvaian MJ, Lee MS, Lee KH, Hernandez S, Kwan A, et al. Safety and clinical activity of $1 \mathrm{~L}$ atezolizumab + bevacizumab in a phase Ib study in hepatocellular carcinoma (HCC). J Clin Oncol. 2017:suppl abstr 4074.

36 Wainberg ZA, Segal NH, Jaeger D, Lee KH, Marshall J, Antonia SJ, et al. Safety and clinical activity of durvalumab monotherapy in patients with hepatocellular carcinoma (HCC). J Clin Oncol. 2017:suppl abstr 4071.

37 Massard C, Gordon MS, Sharma S, Rafii S, Wainberg ZA, Luke J, et al. Safety and Efficacy of Durvalumab (MEDI4736), an AntiProgrammed Cell Death Ligand-1 Immune Checkpoint Inhibitor, in Patients With Advanced Urothelial Bladder Cancer. J Clin Oncol. 2016 Sep;34(26):3119-25.

38 Cheng AL, Kang YK, Lin DY, Park JW, Kudo M, Qin S, et al. Sunitinib versus sorafenib in advanced hepatocellular cancer: results of a randomized phase III trial. J Clin Oncol. 2013 Nov;31(32):4067-75.

39 Johnson PJ, Qin S, Park JW, Poon RT, Raoul JL, Philip PA, et al. Brivanib versus sorafenib as first-line therapy in patients with unresectable, advanced hepatocellular carcinoma: results from the randomized phase III BRISKFL study. J Clin Oncol. 2013 Oct;31(28): 3517-24.

40 Llovet JM, Decaens T, Raoul JL, Boucher E, Kudo M, Chang C, et al. Brivanib in patients with advanced hepatocellular carcinoma who were intolerant to sorafenib or for whom sorafenib failed: results from the randomized phase III BRISK-PS study. J Clin Oncol. 2013 Oct;31(28):3509-16.

41 Zhu AX, Kudo M, Assenat E, Cattan S, Kang YK, Lim HY, et al. Effect of everolimus on survival in advanced hepatocellular carcinoma after failure of sorafenib: the EVOLVE-1 randomized clinical trial. JAMA. 2014 Jul;312(1): 57-67.

42 Zhu AX, Park JO, Ryoo BY, Yen CJ, Poon R, Pastorelli D, et al.; REACH Trial Investigators. Ramucirumab versus placebo as secondline treatment in patients with advanced hepatocellular carcinoma following first-line therapy with sorafenib (REACH): a randomised, double-blind, multicentre, phase 3 trial. Lancet Oncol. 2015 Jul;16(7):859-70. 
43 Chau I, Park JO, Ryoo BY, Yen CJ, Poon R, Pastorelli D, et al. Alpha-fetoprotein kinetics in patients with hepatocellular carcinoma receiving ramucirumab or placebo: an analysis of the phase 3 REACH study. Br J Cancer. 2018 Jul;119(1):19-26.

44 Smyth MJ, Ngiow SF, Ribas A, Teng MW. Combination cancer immunotherapies tailored to the tumour microenvironment. Nat Rev Clin Oncol. 2016 Mar;13(3):143-58.

45 Domouchtsidou A, Barsegian V, Mueller SP, Best J, Ertle J, Bedreli S, et al. Impaired lymphocyte function in patients with hepatic malignancies after selective internal radiotherapy. Cancer Immunol Immunother. 2018 May; 67(5):843-53.

46 Zhang B, Finn RS. Personalized Clinical Trials in Hepatocellular Carcinoma Based on Biomarker Selection. Liver Cancer. 2016 Jul; 5(3):221-32.

47 Xue R, Li R, Guo H, Guo L, Su Z, Ni X, et al. Variable Intra-Tumor Genomic Heterogeneity of Multiple Lesions in Patients With Hepatocellular Carcinoma. Gastroenterology. 2016 Apr;150(4):998-1008.

48 Chau I, Peck-Radosavljevic M, Borg C, Malfertheiner P, Seitz JF, Park JO, et al. Ramucirumab as second-line treatment in patients with advanced hepatocellular carcinoma following first-line therapy with sorafenib: patient-focused outcome results from the randomised phase III REACH study. Eur J Cancer. 2017 Aug;81:17-25.

49 Chau I, Peck-Radosavljevic M, Borg C, Malfertheiner P, Seitz JF, Park JO, et al. Corrigendum to "Ramucirumab as second-line treatment in patients with advanced hepatocellular carcinoma following first-line therapy with sorafenib: patient-focused outcome results from the randomised phase III REACH study" [Eur J Canc 81 (2017) 17-25]. Eur J Cancer. 2018 Sep;100:135-6.

50 Wang X, Teng F, Kong L, Yu J. PD-L1 expression in human cancers and its association with clinical outcomes. OncoTargets Ther. 2016 Aug;9:5023-39.

51 Kim ST, Klempner SJ, Park SH, Park JO, Park YS, Lim HY, et al. Correlating programmed death ligand 1 (PD-L1) expression, mismatch repair deficiency, and outcomes across tumor types: implications for immunotherapy. Oncotarget. 2017 Aug;8(44):77415-23.

52 Brahmer JR, Drake CG, Wollner I, Powderly JD, Picus J, Sharfman WH, et al. Phase I study of single-agent anti-programmed death-1 (MDX-1106) in refractory solid tumors: safe- ty, clinical activity, pharmacodynamics, and immunologic correlates. J Clin Oncol. 2010 Jul;28(19):3167-75.

53 Reig M, Torres F, Rodriguez-Lope C, Forner A, LLarch N, Rimola J, et al. Early dermatologic adverse events predict better outcome in HCC patients treated with sorafenib. J Hepatol. 2014 Aug;61(2):318-24.

54 Pellino A, Loupakis F, Cadamuro M, Dadduzio V, Fassan M, Guido M, et al. Precision medicine in cholangiocarcinoma. Transl Gastroenterol Hepatol. 2018 Jul;3:40.

55 Edge SB, Compton CC. The American Joint Committee on Cancer: The 7th edition of the AJCC cancer staging manual and the future of TNM. Ann Surg Oncol. 2010 Jun;17(6):14714.

56 Morizane C, Ueno M, Ikeda M, Okusaka T, Ishii H, Furuse J. New developments in systemic therapy for advanced biliary tract cancer. Jpn J Clin Oncol. 2018 Aug;48(8):70311.

57 Zhu Y, Wang XY, Zhang Y, Xu D, Dong J, Zhang $Z$, et al. Programmed death ligand 1 expression in human intrahepatic cholangiocarcinoma and its association with prognosis and CD8+ T-cell immune responses. Cancer Manag Res. 2018 Oct;10:4113-23. 